PROCEEDINGS OF THE

AMERICAN MATHEMATICAL SOCIETY

Volume 125, Number 11, November 1997, Pages 3331-3337

S 0002-9939(97)03954-3

\title{
INFINITE TAYLOR INTERPOLATION
}

\author{
LEONHARD FRERICK AND JÜRGEN MÜLLER
}

(Communicated by Theodore W. Gamelin)

\begin{abstract}
Let $G$ be a region in $\mathbb{C}$, let $\alpha$ be a point in $G$, and let $\Lambda$ be an infinite set of nonnegative integers. We consider the question whether there exists a function which is holomorphic in $G$ and has prescribed derivatives of order $\nu$ at $\alpha$ for all $\nu \in \Lambda$.
\end{abstract}

\section{INTRODUCTION}

Let $G \subset \mathbb{C}$ be a region and let $H(G)$ denote the set of all functions which are holomorphic in $G$. A fundamental result in constructive function theory is the following Hermite interpolation theorem. Let a set $A \subset G$ without accumulation point in $G$ and nonnegative integers $m(\alpha)$ for $\alpha \in A$ be given. Then for every matrix of complex numbers $\left(a_{\nu, \alpha}\right)_{\nu=0, \ldots, m(\alpha) ; \alpha \in A}$ there exists a function $f \in H(G)$ such that

$$
\frac{f^{(\nu)}(\alpha)}{\nu !}=a_{\nu, \alpha} \quad(\nu=0, \ldots, m(\alpha) ; \alpha \in A)
$$

(see for example [7, Theorem 15.13]). The question arises whether for some $\alpha$ the finite sequence $\left(a_{0, \alpha}, \ldots, a_{m(\alpha), \alpha}\right)$ may be replaced by a sequence $\left(a_{\nu}, \nu \in \Lambda\right)$, where $\Lambda \subset \mathbb{N}_{0}$ is infinite and $\mathbb{N}_{0}$ denotes the set of nonnegative integers. Thus, the question is, roughly speaking, whether and to what extent we may prescribe an infinity of derivatives at some point $\alpha$ in $G$. Our aim is to give a partial answer to this question for the case of just one interpolation node $A=\{\alpha\}$, which we suppose to be $\alpha=0$.

If $\Lambda \subset \mathbb{N}_{0}$ is infinite, then of course we have to restrict the sequence $\left(a_{\nu}, \nu \in \Lambda\right)$ in such a way that

$$
\limsup _{\nu \rightarrow \infty, \nu \in \Lambda}\left|a_{\nu}\right|^{1 / \nu} \leq \frac{1}{\operatorname{dist}(0, \partial G)} .
$$

We call such a sequence admissible. Since the problem turns out to be trivial for $G=\mathbb{C}$ (every admissible sequence obviously works), we may suppose $\operatorname{dist}(0, \partial G)=$ 1. Now our problem on Taylor interpolation reads as follows.

Let $G \subset \mathbb{C}$ be a region with $\mathbb{D}:=\{z:|z|<1\} \subset G$ and $\overline{\mathbb{D}} \not \subset G$. Which (infinite) sets $\Lambda \subset \mathbb{N}_{0}$ have the property that for every admissible sequence $\left(a_{\nu}, \nu \in \Lambda\right)$ there

Received by the editors March 1, 1996 and, in revised form, June 14, 1996.

1991 Mathematics Subject Classification. Primary 30E05.

(C)1997 American Mathematical Society 
exists a function $f \in H(G)$ such that

$$
\frac{f^{(\nu)}(0)}{\nu !}=a_{\nu} \quad(\nu \in \Lambda)
$$

which means

$$
f(z)=\sum_{\nu \in \Lambda} a_{\nu} z^{\nu}+\sum_{\nu \in \Lambda^{\prime}} a_{\nu} z^{\nu} \quad(|z|<1)
$$

for a suitable sequence $\left(a_{\nu}, \nu \in \Lambda^{\prime}\right), \Lambda^{\prime}:=\mathbb{N}_{0} \backslash \Lambda$ ?

We say that $\Lambda$ is $G$-interpolating if (1) holds for all admissible $\left(a_{\nu}, \nu \in \Lambda\right)$. Obviously, $\Lambda$ is $G$-interpolating if $\Lambda$ is finite, and $\Lambda$ is not $G$-interpolating if $\mathbb{N}_{0} \backslash \Lambda$ is finite (and $G \neq \mathbb{D}$ ). But how about other sets $\Lambda$ ? It is clear that the chance for $\Lambda$ to be $G$-interpolating is greater the smaller $\Lambda$ is.

The key observation for everything that follows is a kind of duality between the interpolation problem (1) and classical results concerning singularities of lacunary power series. To make this more precise we have to introduce some notation.

For a subset $M$ of the extended plane $\mathbb{C}_{\infty}:=\mathbb{C} \cup\{\infty\}$ let $H(M)$ denote the set of germs of functions holomorphic on $M$, i.e. $H(M)$ is the set of (equivalence classes of) functions $\varphi: U \rightarrow \mathbb{C}$ where $\varphi$ is holomorphic on some open set $U=U(\varphi) \supset M$ and $\varphi_{1} \sim \varphi_{2}$ if there exists an open set $U \supset M$ such that $U\left(\varphi_{j}\right) \supset U$ for $j=1,2$ and $\varphi_{1 \mid U}=\varphi_{2 \mid U}$. (A function $\varphi$ is said to be holomorphic at $\infty$ if $\varphi(1 / z)$ is holomorphic at zero.)

If we, as usual, endow $H(G)$ with the topology of locally uniform convergence, then $H(G)$ is a Fréchet space and the dual $H(G)^{\prime}$ may be identified with $H\left(G^{\prime}\right)$, where $G^{\prime}:=\mathbb{C}_{\infty} \backslash G$. We restrict ourselves to simply connected regions $G$. Then $U(\varphi)$ may always be assumed to be a simply connected region in $\mathbb{C}_{\infty}$ and the duality is for $f \in H(G)$ and $\varphi \in H\left(G^{\prime}\right)$ given by

$$
\langle f, \varphi\rangle:=\frac{1}{2 \pi i} \int_{\Gamma} f(\zeta) \varphi(\zeta) \frac{d \zeta}{\zeta},
$$

where $\Gamma \subset G \cap U(\varphi)$ is a positively oriented, smooth Jordan curve containing the origin in its interior (see for example [3, p. 55]). By a standard region $G$ we understand in the sequel a simply connected region $G \subset \mathbb{C}$ such that $\operatorname{dist}(0, \partial G)=1$ and $\mathbb{D} \neq G$ (in the case $G=\mathbb{D}$, every admissible sequence works). For a set $\Lambda \subset \mathbb{N}_{0}$ we define $\Lambda^{\prime}:=\mathbb{N}_{0} \backslash \Lambda$ and

$$
H_{\Lambda}(G):=\left\{f \in H(G): f(z)=\sum_{\nu \in \Lambda} a_{\nu} z^{\nu} \text { for } z \in \mathbb{D}\right\}
$$

as well as

$$
H_{\Lambda}\left(G^{\prime}\right):=\left\{\varphi \in H\left(G^{\prime}\right): \varphi(z)=\sum_{\nu \in \Lambda} \varphi_{\nu} / z^{\nu} \text { for }|z| \text { large enough }\right\} .
$$

Finally, for a subspace $F$ of $H(G)$ let

$$
F^{o}:=\left\{\varphi \in H\left(G^{\prime}\right):\langle f, \varphi\rangle=0 \text { for all } f \in F\right\}
$$

be the polar of $F$. In the next section we present the main results. Auxilliary results and the proofs are given in Section 3. 


\section{RESUlts}

The following is the key duality result.

Theorem 1. Let $G \subset \mathbb{C}$ be a standard region and let $\Lambda$ be a set of nonnegative integers. Then the following assertions are equivalent:

(i) The set $\Lambda$ is $G$-interpolating and $H_{\Lambda^{\prime}}(G)^{\circ}=H_{\Lambda}\left(G^{\prime}\right)$.

(ii) Every $\varphi \in H_{\Lambda}\left(G^{\prime}\right)$ is holomorphic on $\mathbb{D}^{\prime}=\mathbb{C}_{\infty} \backslash \mathbb{D}$, that is,

$$
H_{\Lambda}\left(G^{\prime}\right)=H_{\Lambda}\left(\mathbb{D}^{\prime}\right)
$$

As is easily seen, condition (ii) is an assertion on singularities of lacunary power series. Moreover, the next theorem (which may be viewed as an extension of the classical Runge approximation theorem corresponding to the case $\Lambda=\emptyset$ ) shows that the condition $H_{\Lambda^{\prime}}(G)^{o}=H_{\Lambda}\left(G^{\prime}\right)$ turns out to be a result on lacunary polynomial approximation. We define for $\Sigma \subset \mathbb{N}_{0}$

$$
\Pi_{\Sigma}:=\operatorname{linspan}\left\{z^{\nu}: \nu \in \Sigma\right\},
$$

the set of polynomials having exponents only in $\Sigma$.

Theorem 2. Let $G \subset \mathbb{C}$ be a standard region and let $\Lambda$ be a set of nonnegative integers. Then $H_{\Lambda^{\prime}}(G)^{o}=H_{\Lambda}\left(G^{\prime}\right)$ if and only if $H_{\Lambda^{\prime}}(G)=\overline{\Pi_{\Lambda^{\prime}}}$.

Thus, we have a mutual relation between (1), singularities of lacunary power series and lacunary polynomial approximation. Since $H_{\Lambda^{\prime}}(G)=\overline{\Pi_{\Lambda^{\prime}}}$ is always satisfied if $G$ is starlike with respect to the origin (see for example [1, Theorem 1]), we obtain

Corollary 1. Let $G$ be a standard region starlike with respect to the origin. Then $\Lambda$ is $G$-interpolating if and only if $H_{\Lambda}\left(G^{\prime}\right)=H_{\Lambda}\left(\mathbb{D}^{\prime}\right)$.

Theorem 1 and Corollary 1 make it possible to apply some deep results on singularities of lacunary power series, namely the Pólya gap theorem, which is an extension of Fabry's gap theorem, and the converse of Pólya's theorem proved by Fuchs (cf. [6, p. 8]). In order to formulate the results, one has to invent (as Pólya originally did) the maximal density of a set $\Lambda \subset \mathbb{N}_{0}$.

Definition. For $\Lambda \subset \mathbb{N}_{0}$ let $n_{\Lambda}(r)$ be the number of elements of $\Lambda \cap[0, r]$. Then

$$
d_{\max }(\Lambda):=\lim _{\mu \rightarrow 1^{-}}\left(\limsup _{r \rightarrow \infty} \frac{n_{\Lambda}(r)-n_{\Lambda}(\mu r)}{(1-\mu) r}\right)
$$

is called the maximum density of $\Lambda$.

Remark. If $\Lambda$ is measurable, that is,

$$
d(\Lambda):=\lim _{r \rightarrow \infty} \frac{n_{\Lambda}(r)}{r},
$$

the density of $\Lambda$, exists, then it is easily seen that $d_{\max }(\Lambda)=d(\Lambda)$. Moreover, for arbitrary $\Lambda$, the maximum density exists, and $d_{\max }(\Lambda)$ equals the minimum over the densities of measurable $\tilde{\Lambda}$ containing $\Lambda$ (cf. [5, p. 178]). In particular, if $d_{\max }(\Lambda)=0$, then $\Lambda$ is measurable and $d(\Lambda)=0$.

As an application of Pólya's theorem we obtain

Theorem 3. Let $d \in[0,1)$ be given and let $G$ be a standard region such that $\Omega:=\left\{z e^{i \varphi}: z \in G ;|\varphi| \leq \pi d\right\}$ is contained in a standard region. If $d_{\max }(\Lambda) \leq d$, then $\Lambda$ is G-interpolating. 
Since for $d=0$ every standard region $G$ satisfies the additional condition of Theorem 3, we get

Corollary 2. If $G$ is a standard region and if $d(\Lambda)=0$, then $\Lambda$ is $G$-interpolating.

Under the conditions of Theorem 3 we have in particular that $G \cap\{|z|=1\}$ is contained in some open arc of length $2 \pi(1-d)$. On the other hand, the converse of Pólya's theorem implies

Theorem 4. Let $G$ be a standard region such that $G \cap\{|z|=1\}$ contains an arc of length $2 \pi(1-d)$ for some $d \in[0,1)$. If $d_{\max }(\Lambda)>d$, then $\Lambda$ is not $G$-interpolating.

Since every standard region contains some subarc of $\{|z|=1\}$, we find

Corollary 3. If $G$ is an arbitrary standard region and if $d_{\max }(\Lambda)=1$, then $\Lambda$ is not $G$-interpolating.

A connection of Theorems 3 and 4 yields in particular

Corollary 4. If the standard region $G$ is starlike with respect to the origin, and if $G \cap\{|z|=1\}$ is an open arc of length $2 \pi(1-d)$ for some $d \in[0,1)$, then $\Lambda$ is $G$-interpolating if and only if $d_{\max }(\Lambda) \leq d$.

The most important examples are given by the regions

$$
G_{d}:=\mathbb{C} \backslash\{z:|z|>1,|\arg (z)| \leq \pi d\} \quad(0 \leq d<1) .
$$

Here we find from Corollary 4 that $\Lambda$ is $G_{d}$-interpolating if and only if $d_{\max }(\Lambda) \leq d$.

Remark. The investigations made above could be continued in several directions. A question which obviously arises is that of prescribing (infinitely or finitely many) derivatives at other interpolation nodes $\alpha$. Even the case of entire functions $(G=\mathbb{C})$ seems to be complicated here. A related problem was studied by Kalton and Rubel in $[2]$.

\section{Auxiliary Results AND Proofs}

The main tool to obtain Theorem 1 consists in a functional analytic reformulation of (1).

Lemma 1. Let $G$ be a standard region and let $\Lambda \subset \mathbb{N}_{0}$ be given. Then the following assertions are equivalent.

(i) The set $\Lambda$ is G-interpolating.

(ii) The linear mapping $T_{\Lambda}: H(G) \rightarrow H(\mathbb{D})$, defined by

$$
T_{\Lambda}(f)(z):=\sum_{\nu \in \Lambda} a_{\nu} z^{\nu} \quad \text { where } f(z)=\sum_{\nu \in \mathbb{N}_{0}} a_{\nu} z^{\nu},
$$

has the range $H_{\Lambda}(\mathbb{D})$.

(iii) The dual mapping $T_{\Lambda}^{\prime}: H\left(\mathbb{D}^{\prime}\right) \rightarrow H\left(G^{\prime}\right)$ has the range $H_{\Lambda^{\prime}}(G)^{o}$.

Proof. Since a sequence $\left(a_{\nu}, \nu \in \Lambda\right)$ is admissible if and only if the power series $g(z)=\sum_{\nu \in \Lambda} a_{\nu} z^{\nu}$ is holomorphic in $\mathbb{D}$, the equivalence of (i) and (ii) follows directly from the definition of $T_{\Lambda}$.

Obviously, $T_{\Lambda}$ is linear. The Cauchy integral formula yields for arbitrary $r<1$ with $s \in(r, 1)$

$$
\max _{|z| \leq r}\left|T_{\Lambda}(f)(z)\right| \leq \frac{s}{s-r} \cdot \max _{|z|=s}|f(z)|
$$


which implies the continuity of $T_{\Lambda}$. Moreover, we find for the null space $N\left(T_{\Lambda}\right)=$ $T_{\Lambda}^{-1}(0)$

$$
N\left(T_{\Lambda}\right)=\left\{f \in H(G): f(z)=\sum_{\nu \in \Lambda^{\prime}} a_{\nu} z^{\nu} \text { for some }\left(a_{\nu}, \nu \in \Lambda^{\prime}\right)\right\}=H_{\Lambda^{\prime}}(G) .
$$

Since $H_{\Lambda}(\mathbb{D})$ is a closed subspace of $H(\mathbb{D})$, the implication (ii) to (iii) follows from the closed range theorem (see for example [4, p. 84]). On the other hand, if (iii) holds, then the closed range theorem implies that the range $R\left(T_{\Lambda}\right)$ of $T_{\Lambda}$ is closed. Thus, it sufficies to show that in this case, $R\left(T_{\Lambda}\right)=H_{\Lambda}(\mathbb{D})$.

Obviously, we always have $R\left(T_{\Lambda}\right) \subset H_{\Lambda}(\mathbb{D})$. But, since $\Pi_{\Lambda}=T_{\Lambda}\left(\Pi_{\Lambda}\right) \subset R\left(T_{\Lambda}\right)$ and since $\overline{\Pi_{\Lambda}}=H_{\Lambda}(\mathbb{D})$, the closedness of $R\left(T_{\Lambda}\right)$ also implies $R\left(T_{\Lambda}\right) \supset H_{\Lambda}(\mathbb{D})$.

Lemma 2. Let $G, \Lambda$ and $T_{\Lambda}$ be as in Lemma 1. Then the dual mapping $T_{\Lambda}^{\prime}$ : $H\left(\mathbb{D}^{\prime}\right) \rightarrow H\left(G^{\prime}\right)$ is given by

$$
T_{\Lambda}^{\prime}(\varphi)(z)=\sum_{\nu \in \Lambda} \varphi_{\nu} / z^{\nu} \quad \text { where } \varphi(z)=\sum_{\nu \in \mathbb{N}_{0}} \varphi_{\nu} / z^{\nu}
$$

In particular, we have $R\left(T_{\Lambda}^{\prime}\right)=H_{\Lambda}\left(\mathbb{D}^{\prime}\right) \subset H_{\Lambda}\left(G^{\prime}\right)$.

Proof. Let $S: H\left(\mathbb{D}^{\prime}\right) \rightarrow H\left(G^{\prime}\right)$ be defined by

$$
S(\varphi):=\sum_{\nu \in \Lambda} \varphi_{\nu} / z^{\nu} \quad \text { where } \varphi(z)=\sum_{\nu \in \mathbb{N}_{0}} \varphi_{\nu} / z^{\nu} .
$$

We have to show that

$$
\left\langle T_{\Lambda}(f), \varphi\right\rangle=\langle f, S(\varphi)\rangle \quad \text { for all } f \in H(G), \varphi \in H\left(\mathbb{D}^{\prime}\right) .
$$

If $\varphi \in H\left(\mathbb{D}^{\prime}\right)$, then for some $r<1$

$$
\varphi(z)=\sum_{\nu \in \mathbb{N}_{0}} \varphi_{\nu} / z^{\nu} \quad \text { uniformly in }|z| \geq r .
$$

According to the Cauchy integral formula, we obtain for $g \in H(\mathbb{D})$ with $g(z)=$ $\sum_{\nu \in \mathbb{N}_{0}} b_{\nu} z^{\nu}$

$$
\langle g, \varphi\rangle=\frac{1}{2 \pi i} \int_{|\zeta|=r} g(\zeta) \varphi(\zeta) \frac{d \zeta}{\zeta}=\sum_{\nu \in \mathbb{N}_{0}} b_{\nu} \frac{1}{2 \pi i} \int_{|\zeta|=r} \zeta^{\nu} \varphi(\zeta) \frac{d \zeta}{\zeta}=\sum_{\nu \in \mathbb{N}_{0}} b_{\nu} \varphi_{\nu} .
$$

This shows that for $f \in H(G)$ with $f(z)=\sum_{\nu=0}^{\infty} a_{\nu} z^{\nu}$

$$
\left\langle T_{\Lambda}(f), \varphi\right\rangle=\sum_{\nu \in \Lambda} a_{\nu} \varphi_{\nu} .
$$

On the other hand, since obviously $R(S) \subset H\left(\mathbb{D}^{\prime}\right)$, we also have

$$
\langle f, S(\varphi)\rangle=\sum_{\nu \in \Lambda} a_{\nu} \varphi_{\nu}
$$

The additional assertion on the range of $T_{\Lambda}^{\prime}$ now follows directly from the explicit representation of $T_{\Lambda}^{\prime}$.

Proof of Theorem 1. 1. We show that (i) implies (ii). Since $\Lambda$ is $G$-interpolating and $H_{\Lambda^{\prime}}(G)^{o}=H_{\Lambda}\left(G^{\prime}\right)$, the dual mapping $T_{\Lambda}^{\prime}$ has range $R\left(T_{\Lambda}^{\prime}\right)=H_{\Lambda}\left(G^{\prime}\right)$ by Lemma 1. From Lemma 2 it therefore follows that $H_{\Lambda}\left(G^{\prime}\right)=H_{\Lambda}\left(\mathbb{D}^{\prime}\right)$. 
2. We show that (ii) implies (i). Obviously, we have $\Pi_{\Lambda^{\prime}} \subset H_{\Lambda^{\prime}}(G)$ and therefore $H_{\Lambda^{\prime}}(G)^{o} \subset \Pi_{\Lambda^{\prime}}^{o}$. Since for every $n \in \mathbb{N}_{0}$ and every $\varphi \in H\left(G^{\prime}\right)$ with $\varphi(z)=$ $\sum_{\nu \in \mathbb{N}_{0}} \varphi_{\nu} / z^{\nu}$

$$
\left\langle z^{n}, \varphi\right\rangle=\frac{1}{2 \pi i} \int_{|\zeta|=r} \zeta^{n} \varphi(\zeta) \frac{d \zeta}{\zeta}=\varphi_{n}
$$

(where $r=r(\varphi)$ is sufficiently large), we find

$$
\Pi_{\Lambda^{\prime}}^{o}=\left\{\varphi \in H\left(G^{\prime}\right):\langle P, \varphi\rangle=0 \text { for every } P \in \Pi_{\Lambda^{\prime}}\right\}=H_{\Lambda}\left(G^{\prime}\right),
$$

and thus $H_{\Lambda^{\prime}}(G)^{o} \subset H_{\Lambda}\left(G^{\prime}\right)$. On the other hand, let $\varphi \in H_{\Lambda}\left(G^{\prime}\right)$ be given. By (ii) we have $\varphi \in H_{\Lambda}\left(\mathbb{D}^{\prime}\right)$, and therefore, according to $(2),\langle f, \varphi\rangle=0$ holds for every $f \in H_{\Lambda^{\prime}}(G)$, which means $\varphi \in H_{\Lambda^{\prime}}(G)^{o}$. This shows $H_{\Lambda}\left(G^{\prime}\right) \subset H_{\Lambda^{\prime}}(G)^{o}$.

It remains to show that $\Lambda$ is $G$-interpolating. By Lemma 1 , this means that $T_{\Lambda}^{\prime}$ has range $H_{\Lambda}\left(G^{\prime}\right)=H_{\Lambda^{\prime}}(G)^{o}$. But, taking Lemma 2 into account, this is true because of condition (ii).

Proof of Theorem 2. As we have seen in the proof of Theorem 1, part 2, we always have $\Pi_{\Lambda^{\prime}}^{o}=H_{\Lambda}\left(G^{\prime}\right)$. Now, if $H_{\Lambda^{\prime}}(G)^{o}=H_{\Lambda}\left(G^{\prime}\right)$, then we get $\Pi_{\Lambda^{\prime}}^{o}=H_{\Lambda^{\prime}}(G)^{o}$. According to the bipolar theorem, we find

$$
\overline{\Pi_{\Lambda^{\prime}}}=\Pi_{\Lambda^{\prime}}^{o o}=H_{\Lambda^{\prime}}(G)^{o o}=\overline{H_{\Lambda^{\prime}}(G)}=H_{\Lambda^{\prime}}(G) .
$$

If, on the other hand, $\overline{\Pi_{\Lambda^{\prime}}}=H_{\Lambda^{\prime}}(G)$, then

$$
H_{\Lambda}\left(G^{\prime}\right)=\Pi_{\Lambda^{\prime}}^{o}={\overline{\Pi_{\Lambda^{\prime}}}}^{o}=H_{\Lambda^{\prime}}(G)^{o} .
$$

Proof of Theorem 3. We show that condition (ii) of Theorem 1 is satisfied. Let therefore $\varphi \in H_{\Lambda}\left(G^{\prime}\right)$ be given, and let $r>0$ be the radius of convergence of the power series

$$
\psi(w):=\varphi(1 / w)=\sum_{\nu \in \Lambda} \varphi_{\nu} w^{\nu}
$$

around the origin. We have to prove that $r>1$.

If $r=\infty$, then nothing has to be done. So we may suppose $r<\infty$. Since $d_{\max }(\Lambda) \leq d$, the Pólya gap theorem (see for example [6, p. 3]) tells us that $\psi$ has a singularity on every closed subarc of $|w|=r$ of length $2 \pi d r$ (and is noncontinuable beyond $|w|=r$ if $d=0$ ). This implies that $\varphi$ must have a singularity on every closed subarc of $|z|=1 / r$ of length $2 \pi d / r$ (and is noncontinuable beyond $|z|=1 / r$ if $d=0$ ). But from our assumptions on $G$ we obtain that $G^{\prime}$ contains some set $\left\{z e^{i \varphi}: z \in \gamma,|\varphi| \leq \pi d\right\}$, where $\gamma$ is a connected and unbounded set such that $\{|z|=1\} \cap \gamma \neq \varnothing$. This forces $1 / r$ to be smaller than 1 .

Remark. Since we have shown that under the assumptions on $G$ and $\Lambda$ made in Theorem 3, the condition (ii) of Theorem 1 is satisfied, we may also conclude that $H_{\Lambda^{\prime}}(G)=\overline{\Pi_{\Lambda^{\prime}}}$ (use Theorem 2 ). This gives a result on lacunary polynomial approximation, which extends the one found in [1, Theorem 5], dealing with the case $d(\Lambda)=0$.

Proof of Theorem 4. Let $\epsilon>0$ be such that

$$
d_{\max }(\Lambda)>d+\epsilon
$$


and let $\Gamma$ be a closed subarc of $G \cap\{|z|=1\}$ of length $2 \pi(1-d-\epsilon)$. Then there exists an $\eta>0$ such that

$$
\tilde{G}:=\mathbb{D} \cup \bigcup_{z \in \Gamma}\{\zeta:|\zeta-z|<\eta\} \subset G .
$$

Obviously, $\tilde{G}$ is a standard region which is starlike with respect to the origin. Since $\tilde{G} \subset G$, it suffices to show that $\Lambda$ is not $\tilde{G}$-interpolating.

Because $\tilde{G}$ is starlike with respect to 0 , we have $H_{\Lambda^{\prime}}(\tilde{G})^{o}=H_{\Lambda}\left(\tilde{G}^{\prime}\right)$ by Theorem 2 and by the result of Dixon and Korevaar ([1, Theorem 1]) cited above. Therefore, according to Theorem 1 , our assertion is equivalent to $H_{\Lambda}\left(\mathbb{D}^{\prime}\right) \neq H_{\Lambda}\left(\tilde{G}^{\prime}\right)$.

By construction, $\tilde{G}^{\prime} \cap\{|z|=1\}$ is contained in an arc $\gamma$ of length $2 \pi(d+\epsilon)$. Since $d_{\max }(\Lambda)>d+\epsilon$, the converse of Pólya's gap theorem (cf. [6, p.8]) shows that there exists a power series

$$
\psi(w)=\sum_{\nu \in \Lambda} \psi_{\nu} w^{\nu}
$$

with radius of convergence 1 and such that $\psi$ has no singularity on $\{w:|w|=$ $1,|\arg (w)| \leq \pi(d+\epsilon)\}$. Thus, for an appropriate $\zeta$ with $|\zeta|=1$, the function

$$
\varphi(z):=\psi(\zeta / z)=\sum_{\nu \in \Lambda} \psi_{\nu} \zeta^{\nu} / z^{\nu}
$$

is holomorphic on $\{|z|>1\} \cup \gamma \supset \tilde{G}^{\prime}$ and has a singularity on $\{|z|=1\}$, which implies

$$
\varphi \in H_{\Lambda}\left(\tilde{G}^{\prime}\right) \backslash H_{\Lambda}\left(\mathbb{D}^{\prime}\right)
$$

\section{ACKNOWLEDGMENT}

The authors want to thank A. Sauer from the University of Duisburg for a helpful remark.

\section{REFERENCES}

1. M. Dixon, J. Korevaar, Approximation by lacunary polynomials, Indag. Math., 39 (1977), 176-194. MR 56:15940

2. N. Kalton, L. A. Rubel, Gap-interpolation theorems for entire functions, J. Reine Angew. Math, 316 (1980), 71-82. MR 83f:30023

3. C. A. Berenstein, R. Gay, Complex analysis and special topics in harmonic analysis, Springer, New York, 1995. MR 96j:30001

4. G. Köthe, Topological vector spaces, II, Springer-Verlag, New York, 1979. MR 81g:46001

5. P. Koosis, The logarithmic integral, I, Cambridge University Press, Cambridge, 1988. MR 90a:30097

6. P. Koosis, The logarithmic integral, II, Cambridge University Press, Cambridge, 1992. MR 94i: 30027

7. W. Rudin, Real and complex analysis, 3rd ed., McGraw - Hill, New York, 1987. MR 88k:00002

Bergische Universität Gesamthochschule Wuppertal, Fachbereich 7, Mathematik, 42097 Wuppertal, Germany

E-mail address: leonhard.frerick@math.uni-wuppertal.de

Universität Trier, Fachbereich IV, Mathematik, 54286 Trier, Germany

E-mail address: jmueller@uni-trier.de 